\title{
Development of a real-time quantitative PCR assay for detection of a stable genomic region of BK virus
}

\author{
Kosuke K Iwaki ${ }^{*}$, Suhail H Qazi ${ }^{1}$, Jean Garcia-Gomez ${ }^{1}$, Deanna Zeng ${ }^{1}$, Yasuhiro Matsuda', Kazuko Matsuda', \\ Monica E Martinez ${ }^{1}$, Mieko Toyoda ${ }^{2}$, Arputharaj Kore ${ }^{3}$, Wesley T Stevens ${ }^{4}$, Miroslaw Smogorzewski ${ }^{5}$, \\ Daisuke D Iwaki ${ }^{1}$, Yasir Qazi ${ }^{5}$, Yuichi Iwaki
}

\begin{abstract}
Background: BK virus infections can have clinically significant consequences in immunocompromised individuals. Detection and monitoring of active BK virus infections in certain situations is recommended and therefore PCR assays for detection of BK virus have been developed. The performance of current BK PCR detection assays is limited by the existence of viral polymorphisms, unknown at the time of assay development, resulting in inconsistent detection of $B K$ virus. The objective of this study was to identify a stable region of the BK viral genome for detection by PCR that would be minimally affected by polymorphisms as more sequence data for BK virus becomes available.

Results: Employing a combination of techniques, including amino acid and DNA sequence alignment and interspecies analysis, a conserved, stable PCR target region of the BK viral genomic region was identified within the VP2 gene. A real-time quantitative PCR assay was then developed that is specific for BK virus, has an analytical sensitivity of 15 copies/reaction (450 copies $/ \mathrm{ml}$ ) and is highly reproducible (CV $\leq 5.0 \%)$.

Conclusion: Identifying stable PCR target regions when limited DNA sequence data is available may be possible by combining multiple analysis techniques to elucidate potential functional constraints on genomic regions. Applying this approach to the development of a real-time quantitative PCR assay for BK virus resulted in an accurate method with potential clinical applications and advantages over existing BK assays.
\end{abstract}

\section{Background}

BK virus (BKV), along with JC virus (JCV) and Simian virus 40 (SV40), are members of the family Polyomaviridae. BKV and JCV are ubiquitous in human populations worldwide with a seroprevalence in adults of $70 \%-80 \%$ [1-4]. They establish persistent, latent infections and are capable of reactivating in immunosuppressed hosts [5-7]. BKV in particular is recognized as a significant cause of allograft failure in renal transplant recipients [8]. In addition, these viruses may also be associated with renal dysfunction in nonrenal transplant recipients $[9,10]$. Prospective monitoring of patients at risk for BKV-associated nephropathy (BKVAN) or BKV associated morbidity may identify those patients with active infection before renal function deteriorates [11-13]. Early identification of active BK infection in transplant recipients is advantageous for

\footnotetext{
* Correspondence: keniwaki@metic.com

'Metic Transplantation Laboratory, USC, Keck School of Medicine, Los Angeles, CA, USA

Full list of author information is available at the end of the article
}

controlling BKV replication and preventing BKVAN via reduction of immunosuppression or use of cidofovir antiviral therapy $[14,15]$.

BKV screening protocols and quantitative BKV testing are increasingly performed in molecular virology laboratories. Guidelines for quantitative cutoffs for nucleic acid tests for BK viruria and BK viremia that indicate the need for additional clinical testing were proposed in 2005 [5]. However, the usefulness of these cutoffs is hampered by the lack of standardized assays, uniform external viral standard, the existence of viral subtypes and the presence of viral polymorphisms. Primers and probes developed for quantitative BKV testing based on limited available sequence data from few viral isolates suffer reduced performance in detection of viral isolates with sequence variations in these regions. This in turn can lead to inconsistent detection of virus, inaccurate quantitation of viral load and difficulty comparing results between assays.
C Biomed Central

() 2010 Iwaki et al; licensee BioMed Central Ltd. This is an Open Access article distributed under the terms of the Creative Commons Attribution License (http://creativecommons.org/licenses/by/2.0), which permits unrestricted use, distribution, and reproduction in any medium, provided the original work is properly cited. 
We describe an approach for the development of a stable nucleic acid assay when limited nucleotide sequence information is available. We report that interspecies amino acid and nucleotide sequence analysis, in conjunction with intraspecies nucleotide sequence alignment, can elucidate genomic regions that may be under potential functional constraints and that these regions can be targeted for primer and probe design to improve assay performance.

\section{Results}

Intraspecies nucleotide sequence variation analysis of BK viral genes

The initial step in identifying a stable region of the BK viral genome for assay development was nucleotide sequence variation analysis of the BK virus (BKV) genes, performed on 157 to $160 \mathrm{BKV}$ isolates using ClustalW2 [16] and MUSCLE [17] analyses. The total number of polymorphisms per gene based on multiple nucleotide sequence alignments for the six BKV genes is given in Table 1 . These analyses show that sequence variants are widespread throughout the BKV genome and occur in all 6 viral genes. Of note, in comparison to most other areas of the BKV genome, there were substantially fewer sequence variants corresponding to the $\mathrm{C}$-terminus of the VP2 gene. The distribution of sequence variants in the C-terminus region of the VP2 is presented in Figure 1.

Several regions of the BKV genome demonstrated either $100 \%$ homology or limited to minor mismatches among the BKV isolates being compared. These regions, including sections of the $\mathrm{C}$-terminal helicase domain of the Large- $\mathrm{T}$ antigen, sections of the $\mathrm{N}$-terminal heat shock protein DNA-J of the Large-T antigen, sections of the N-terminus of the VP2 gene, and sections of the Cterminus of the VP2/VP3 gene, were considered equally suitable targets for assay development at this stage.

Interspecies amino acid analysis of potential assay target regions in Polyomaviridae family members

To further evaluate the potential assay target regions identified by intraspecies nucleotide analysis and to

Table 1 Polymorphisms in BKV Genes

\begin{tabular}{ccc}
\hline & \# of SNPs* & $\begin{array}{c}\text { Gene size } \\
\text { (bp) }\end{array}$ \\
\hline Agno protein & 48 & 201 \\
Small - T antigen & 53 & 519 \\
Large - T antigen & 316 & 2088 \\
VP - 1 & 179 & 1089 \\
VP - 2 & 106 & 1056 \\
VP - 3 & 86 & 699 \\
\hline
\end{tabular}

*ClustalW2 alignment of available nucleotide sequences in GENBANK. The size of each respective gene is based on the NCBI reference sequence (accession number: NC_001538). develop supporting evidence for the sequence stability of these regions, an interspecies amino acid sequence comparison of each of the regions was performed. Among the four potential target regions, the VP2 C-terminus region was distinguished from the others due to the presence of a number of motifs and recognized structural elements including: (1) an alpha helix at the C-terminus [18], (2) a known VP1 interaction region in the C-terminus [18], (3) a series of basic amino acid residues comprising a nuclear localization signal (NLS) [19-22], (4) a DNA binding region $[23,24]$ and (5) a shared open reading frame due to the overlap with the N-terminus of VP1 $[25,26]$. Examination of members of the Polyomaviridae family revealed that the VP2 C-terminus region and the alphahelical region in particular, are conserved across multiple members of the Polyomaviridae family, suggestive of a region under a high degree of structural and functional constraint (Figure 2). It also confirmed previous observations [18] that the residues downstream of the alphahelix region exhibit more variability across members of the Polyomaviridae family. Lastly, members of the Polyomaviridae family that are more closely related to BKV (i.e. JCV, Simian Agent [SA]12, and SV40) exhibit stretches of amino acids downstream of the alpha-helical region that are also conserved (Figure 2). The degree of sequence conservation in the VP2 C-terminus region and the presence of known structural and functional elements suggest a reduced likelihood of sequence variation. In addition, a section of the VP2 C-terminus, (residues 324330, SRGSSQK), is conserved in BKV, SV40, and SA12, but absent in JCV. Taken together, these results support the C-terminus of VP2 as a sequence stable target for assay development that could also be specific for BKV, relative to JCV.

\section{Interspecies and intraspecies comparison of nucleotide sequences of the assay target region}

The regions of the VP2 C-terminus identified by amino acid alignment were further analyzed by intraspecies sequence analysis of JCV isolates and interspecies nucleotide sequence alignment among very closely related viruses (Figure 1). This enabled cross species comparison of the relative locations of polymorphisms within the selected target region. Focus was primarily directed at comparing the BKV and JCV sequences as they are clinically important viruses that are taxonomically closely related and there were a considerably greater number of sequences available for JCV.

Intraspecies nucleotide sequence analysis of the VP2 gene identified several stretches of conserved codons among BKV isolates (Figure 1). The nucleotide sequence 1 to 135 (residues 272-316, Figure 2) is conserved among BKV sequences, containing only three polymorphic positions. These polymorphisms represent synonymous 


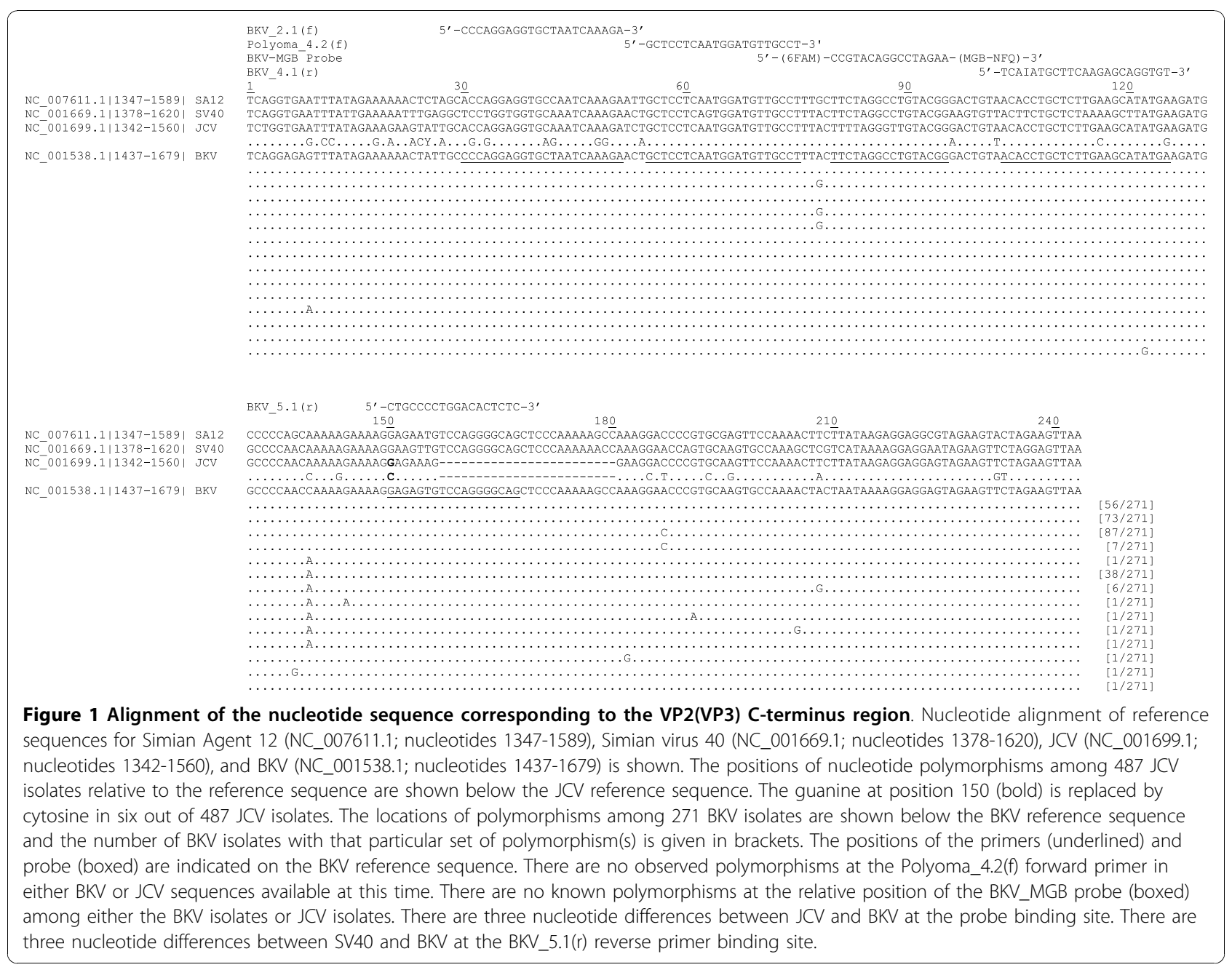

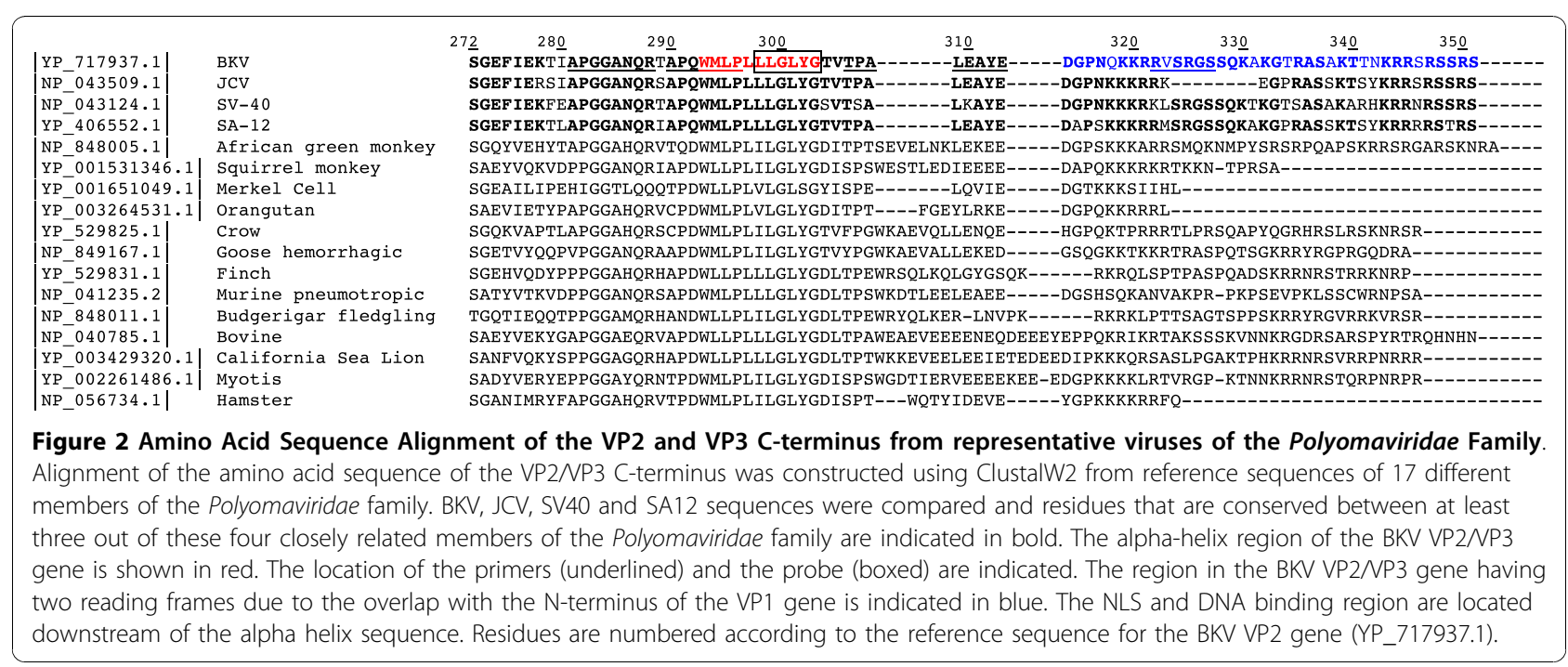


mutations at Glu (residue 274) and Leu (residue 297) and a non-synonymous mutation at residue 312 (Tyr to Cys). Two other stretches of conserved nucleotides were observed: the stretch of 36 nucleotides 145 to 180 corresponding to KRRVSRGSSQKA (residues 320-331, Figure 2) and the stretch of 33 nucleotides 211 to 243 corresponding to NKRRSRSSR (residues 342-351, Figure 2) and stop codon. Polymorphisms were not observed among available BKV sequences for these latter two regions.

Interspecies nucleotide comparison of known polymorphic sites in VP2 between BKV and JCV revealed a stretch of 41 nucleotides 55 to 95 that is devoid of polymorphisms aside for a single polymorphism for BKV at nucleotide 78. This stretch of nucleotides corresponds to the alpha-helix region and the three adjacent up-stream residues APQWMLPLLLGLYG (residues 290-303, Figure 2). With the exception of a single nucleotide polymorphism at position 9 , the sequence upstream of nucleotide position 55 is conserved among BKV sequences. However, a significant number of polymorphic sites are observed among the corresponding JCV nucleotide sequences.

Based on these results, primers and probe were designed targeting the nucleotide sequence corresponding to the VP2 C-terminus region (Figure 1).

\section{Design of PCR assay primers and probe}

The assay forward primer (Polyoma_4.2 [sense strand]) was designed to correspond to nucleotides 55 to 75 of BKV sequence (Figure 1), which contains the codons for part of the alpha-helix region. No polymorphisms were observed within BKV or JCV or between BKV and JCV sequences. An alternate forward primer (BKV_2.1 [sense strand]) was designed corresponding to nucleotides 30 to 51 of BKV sequence (Figure 1). This sequence reflects a region of interspecies amino acid conservation among BKV, JCV, SV40 and SA12 reference strains (residues 281 to 288 , Figure 2). Although the nucleotide analyses indicate it is conserved among the available BKV isolates, this stretch of nucleotides display multiple polymorphisms among the available JCV sequences.

The assay reverse primer (BKV_5.1 [anti-sense strand]) was designed to correspond to nucleotide sequences 150 to 167 of the BKV sequence (Figure 1). This sequence contains the codons for amino acid residues SRGS (residues 324-327, Figure 2) that are deleted in JCV. No polymorphisms are observed along this stretch of nucleotides among the available BKV sequences. Three consecutive nucleotide differences exist between the BKV and SV40 reference sequences within the primer (Figure 1). An alternate reverse primer (BKV_4.1 [anti-sense strand]) was designed corresponding to nucleotides 103 to 125 of the BKV sequence (Figure 1). There is a single polymorphism within BKV isolates in the primer sequence, resulting in use of the universal deoxyinosine nucleotide at the corresponding position in the primer. Several polymorphisms were observed between BKV and JCV reference sequences corresponding to this primer.

The probe sequence (BKV-MGB Probe [anti-sense strand]) corresponds to nucleotide sequences 80 to 95 (Figure 1). This sequence contains the codons for the alpha-helix residues LLGLYG, conserved in the SA12, SV40, JCV and BKV (Figure 2). There are a total of three nucleotide differences between BKV and JCV reference sequences at the probe binding site (Figure 1) and this difference is present in all BKV and JCV isolates. No polymorphisms in the probe sequence were observed between BKV and SV40 reference sequences.

The nucleotide comparisons described above allowed design of a combination of primers and probes that targets a sequence stable region yet provides specificity for $\mathrm{BKV}$ relative to JCV and SV40.

\section{Development and validation of a real-time quantitative PCR assay for the VP2 region of BK virus}

Once a stable region of the BKV genome was identified, a real-time quantitative PCR assay was developed. Serial dilution experiments were undertaken to evaluate assay performance. The BKV assay was linear over a 6-log range from $10^{7}$ copies/reaction to $10^{1}$ copies/reaction (Figure 3).

The sensitivity of the BKV assay was evaluated by testing a BKV standard (Advanced Biotechnologies Inc., MD) at known concentrations of 1.5, 15, 75 and 150 copies/ reaction. Analytical sensitivity was determined by the lowest serial dilution consistently detectable in replicate reactions. The assay detected BKV with 100\% (30/30) sensitivity at BKV copy levels of 15 copies/reaction and greater (Table 2). BKV at a copy level of 1.5 copies/reaction was detected in 2 to 3 of 10 replicates (20-30\%). Therefore the analytical sensitivity of the assay is 15 copies/reaction, which is equivalent to 450 copies $/ \mathrm{ml}$ given a $200 \mu \mathrm{l}$ extraction volume and an elution volume of $60 \mu \mathrm{l}$. The lower limit of detection of the assay, which is detectable but not reliably quantifiable, is 1.5 copies/ reaction.

The specificity of the BKV assay was evaluated by testing replicates of JCV DNA standard (Advanced Biotechnologies) at concentrations of $1.4 \times 10^{4}$ copies/ reaction and mixed samples containing $\mathrm{BKV}$ at various concentrations combined with JCV at $7 \times 10^{3}$ copies/ reaction compared to the same concentrations of $\mathrm{BKV}$ without any JCV present. No false positive results $(0 / 10)$ were observed when testing JCV samples (Table 3). In addition, no inhibition of BKV detection in the presence of JCV was observed, as crossing point (Cp) values for $\mathrm{BKV}$ in mixed samples with JCV were similar for the same concentrations of BKV alone. 
A.

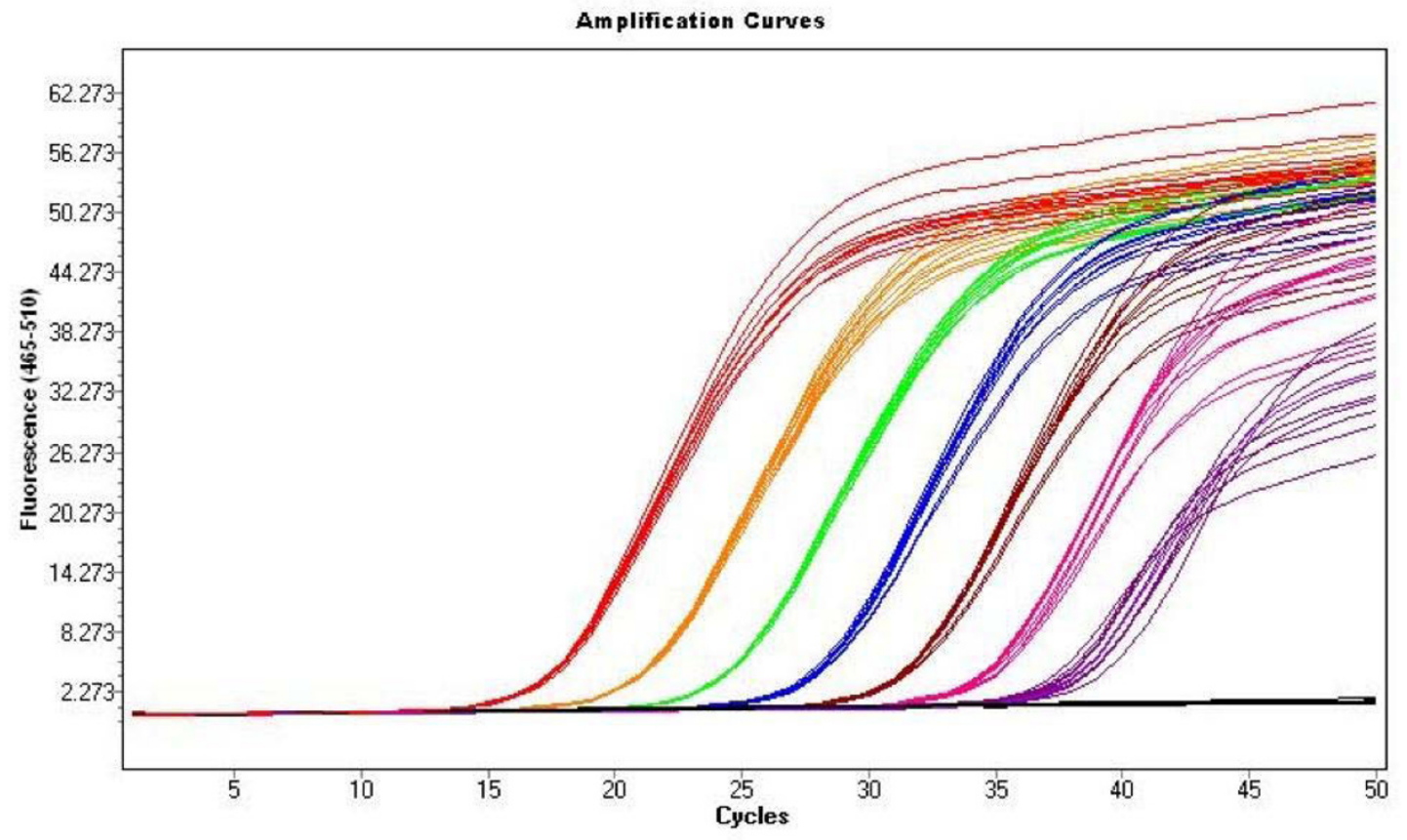

B.

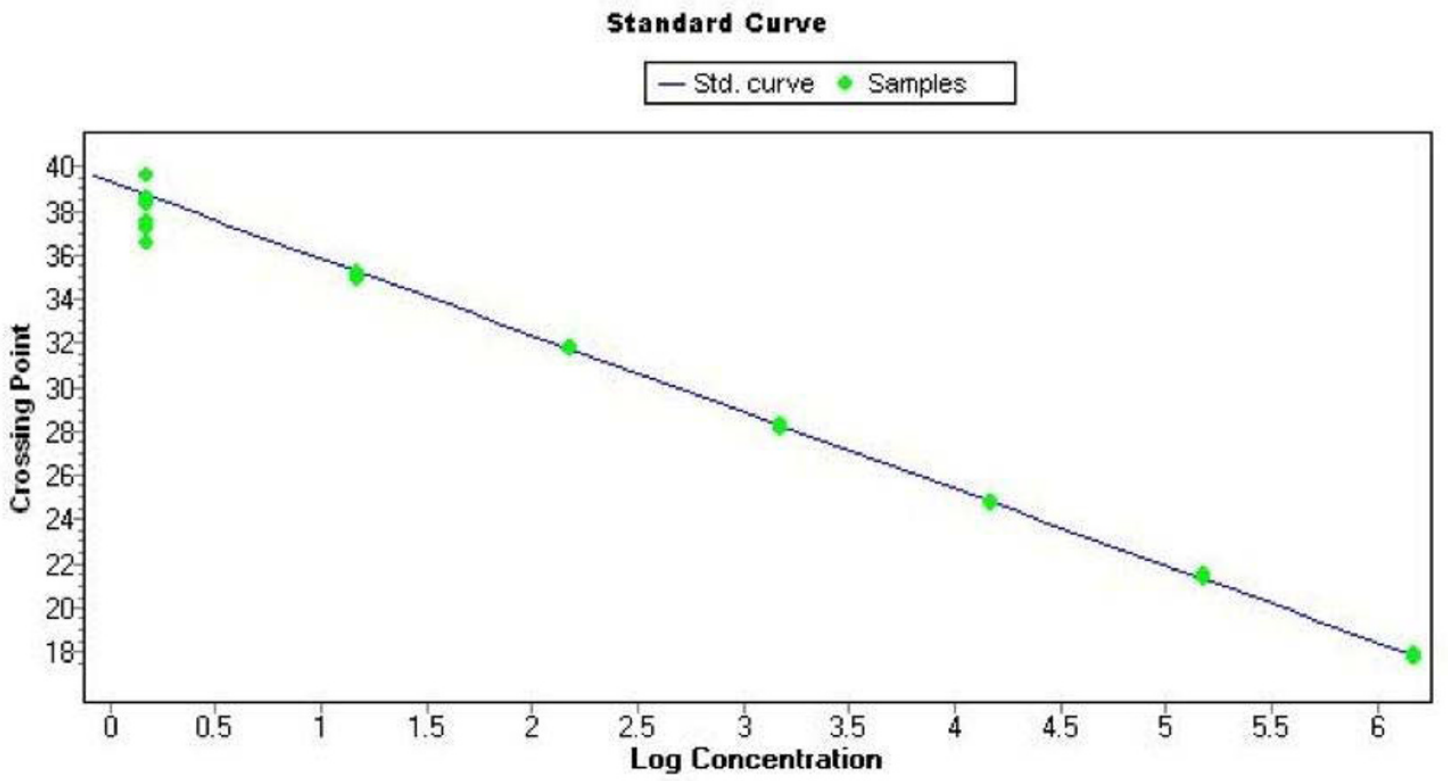

Figure 3 BKV Real-Time Quantitative PCR. The BKV standard was serially diluted prior to being assayed by real-time quantitative PCR. A. Raw amplification curves from precision study with primers BKV_5.1(r) and BKV_4.2(f). Input BKV DNA was $10 \mu \mathrm{l}$ at concentrations of $10^{6}$ copies/ $\mu$, $10^{5}$ copies $/ \mu \mathrm{l}, 10^{4}$ copies $/ \mu \mathrm{l}, 10^{3}$ copies $/ \mu \mathrm{l}, 10^{2}$ copies $/ \mu \mathrm{l}, 10 \mathrm{copies} / \mu \mathrm{l}$ and $<10$ copies $/ \mu \mathrm{l}$. B. Standard curve. Results of the regression were: slope -3.484 , $y$-intercept 39.05 and efficiency 1.936. Similar results were obtained with other primer combinations. 
Table 2 Sensitivity of the BKV Real-Time Quantitative PCR Assay

\begin{tabular}{|c|c|c|c|c|c|c|c|c|c|c|}
\hline \multirow[b]{3}{*}{ Copies per Reaction* } & \multirow[b]{3}{*}{ \# of Reactions } & \multicolumn{9}{|c|}{ Crossing Point } \\
\hline & & \multicolumn{3}{|c|}{ BKV_5.1/Polyoma_4.2 } & \multicolumn{3}{|c|}{ BKV_4.1/Polyoma_4.2 } & \multicolumn{3}{|c|}{ BKV_4.1/BKV_2.1 } \\
\hline & & Mean & SD & CV & Mean & SD & CV & Mean & SD & CV \\
\hline 150 & 10 & 36.16 & 0.75 & 2.06 & 36.46 & 0.33 & 0.90 & 37.19 & 0.31 & 0.82 \\
\hline 75 & 10 & 37.75 & 0.94 & 2.48 & 37.55 & 0.51 & 1.35 & 38.52 & 0.38 & 0.98 \\
\hline 15 & 10 & 39.86 & 1.05 & 2.63 & 39.22 & 0.84 & 2.15 & 40.51 & 1.31 & 3.24 \\
\hline $1.5^{* *}$ & 10 & 40.84 & 0.58 & 1.42 & 41.08 & 0.22 & 0.53 & 43.75 & 2.17 & 4.96 \\
\hline
\end{tabular}

$\mathrm{SD}=$ standard deviation; $\mathrm{CV}$ is a percentage.

*5 replicates of negative control were all negative.

* *only 2 to 3 of 10 replicates for each primer combination had crossing points above background.

The precision of the assay was evaluated based upon the reproducibility of the Cp values for a BKV DNA standard, which was serially diluted to concentrations of $1.5 \times 10^{6}$ copies $/ \mu \mathrm{l}, 1.5 \times 10^{5} \mathrm{copies} / \mu \mathrm{l}, 1.5 \times 10^{4}$ copies $/ \mu \mathrm{l}, 1.5 \times 10^{3}$ copies $/ \mu \mathrm{l}, 1.5 \times 10^{2}$ copies $/ \mu \mathrm{l}, 1.5 \times$ $10^{1}$ copies $/ \mu \mathrm{l}$ and $1.5 \times 10^{0}$ copies $/ \mu \mathrm{l}$. A total of ten replicates for all concentrations were tested. The coefficient of variation $(\mathrm{CV})$ in the $\mathrm{Cp}$ for all concentrations tested was $\leq 2.3 \%$ (Table 4 ), verifying a high degree of assay precision across the entire range of concentrations tested.

\section{Discussion}

There have been previous efforts to develop real-time PCR based BKV quantification assays [27,28]. However, many of these assays were developed when the number of available DNA sequences in public databases was quite limited. Although multiple sequence alignments are routinely used to identify stable target regions for assay development, sequence variants have been observed in these regions as more strains and sequences become available. Therefore, when the number of isolates and sequences is limited, merely selecting a target region for assay development based on intraspecies nucleotide sequence alignments alone may not be sufficiently predictive of interstrain sequence conservation to result in a sensitive and specific assay.

We proposed that identifying areas of the genome that are conserved within and between species could be used to supplement limited nucleotide sequence information when designing assays. Target regions identified via standard intraspecies sequence alignment of full genome sequences from available isolates were further evaluated by interspecies amino acid analysis to select a single target region. The interspecies amino acid alignments assisted in narrowing down potential target regions by identifying areas containing functionally and/or structurally constrained sequences. Conservation in amino acid

Table 3 Specificity of the BKV Real-Time Quantitative PCR Assay

\begin{tabular}{|c|c|c|c|c|c|}
\hline \multirow[b]{3}{*}{ Copies BKV/Reaction* } & \multirow[b]{3}{*}{ Copies JCV/Reaction* } & \multirow[b]{3}{*}{ \# of Reactions } & \multicolumn{3}{|c|}{ Crossing Point } \\
\hline & & & \multicolumn{3}{|c|}{ BKV_5.1/Polyoma_4.2 } \\
\hline & & & Mean & Std Dev & CV (\%) \\
\hline $1.5 \times 10^{7}$ & $7.0 \times 10^{3}$ & 10 & 18.43 & 0.07 & 0.37 \\
\hline $1.5 \times 10^{6}$ & $7.0 \times 10^{3}$ & 10 & 21.99 & 0.02 & 0.11 \\
\hline $1.5 \times 10^{5}$ & $7.0 \times 10^{3}$ & 10 & 25.61 & 0.03 & 0.10 \\
\hline $1.5 \times 10^{4}$ & $7.0 \times 10^{3}$ & 10 & 29.14 & 0.04 & 0.15 \\
\hline $1.5 \times 10^{3}$ & $7.0 \times 10^{3}$ & 10 & 33.06 & 0.08 & 0.25 \\
\hline $1.5 \times 10^{2}$ & $7.0 \times 10^{3}$ & 10 & 36.74 & 0.66 & 1.80 \\
\hline $1.5 \times 10^{1}$ & $7.0 \times 10^{3}$ & 10 & 39.61 & 1.22 & 3.08 \\
\hline $1.5 \times 10^{7}$ & 0 & 2 & 18.41 & 0.02 & 0.12 \\
\hline $1.5 \times 10^{6}$ & 0 & 2 & 22.01 & 0.08 & 0.35 \\
\hline $1.5 \times 10^{5}$ & 0 & 2 & 25.69 & 0.01 & 0.06 \\
\hline $1.5 \times 10^{4}$ & 0 & 2 & 29.28 & 0.08 & 0.27 \\
\hline $1.5 \times 10^{3}$ & 0 & 2 & 33.03 & 0.05 & 0.15 \\
\hline $1.5 \times 10^{2}$ & 0 & 2 & 36.91 & 0.27 & 0.73 \\
\hline $1.5 \times 10^{1}$ & 0 & 2 & 39.94 & 1.97 & 4.94 \\
\hline 0 & $1.4 \times 10^{4}$ & 10 & - & - & - \\
\hline Negative Control & & 5 & - & - & - \\
\hline
\end{tabular}


Table 4 Precision of the BKV Real-Time Quantitative PCR Assay

\begin{tabular}{ccccccccccc}
\hline & & \multicolumn{8}{c}{ Crossing Point } \\
\cline { 3 - 10 } Copies/Reaction* & \# of Reactions & \multicolumn{3}{c}{ BKV_5.1/Polyoma_4.2 } & \multicolumn{1}{c}{ BKV_4.1/Polyoma_4.2 } & \multicolumn{3}{c}{ BKV_4.1/BKV_2.1 } \\
\cline { 2 - 10 } & Mean & CV (\%) & Std Dev & Mean & CV (\%) & Std Dev & Mean & CV (\%) & Std Dev \\
\hline $1.5 \times 10^{7}$ & 10 & 17.77 & 0.30 & 0.05 & 17.68 & 0.89 & 0.16 & 15.89 & 1.33 & 0.21 \\
$1.5 \times 10^{6}$ & 10 & 21.35 & 0.31 & 0.07 & 21.12 & 0.59 & 0.13 & 19.45 & 0.50 & 0.10 \\
$1.5 \times 10^{5}$ & 10 & 24.77 & 0.15 & 0.04 & 24.56 & 0.71 & 0.17 & 22.82 & 1.02 & 0.23 \\
$1.5 \times 10^{4}$ & 10 & 28.25 & 0.21 & 0.06 & 28.09 & 0.43 & 0.12 & 26.27 & 0.72 & 0.19 \\
$1.5 \times 10^{3}$ & 10 & 31.77 & 0.20 & 0.06 & 31.62 & 0.67 & 0.21 & 29.76 & 0.57 & 0.17 \\
$1.5 \times 10^{2}$ & 10 & 35.03 & 0.37 & 0.13 & 35.09 & 0.53 & 0.19 & 33.55 & 0.94 & 0.32 \\
$1.5 \times 10^{1}$ & 10 & 38.03 & 2.30 & 0.88 & 38.41 & 1.50 & 0.58 & 37.33 & 1.79 & 0.67 \\
\hline
\end{tabular}

*5 replicates of negative control were all negative.

sequence among closely related viruses does not necessarily preclude the possibility of non-synonymous substitutions. Functional and structural constraints, however, do suggest that there would likely be fewer number of amino acid residues that can reside at that particular position than if the same region was under less stringent functional or structural constraint.

However, mutations in the nucleotide sequences giving rise to synonymous substitutions can potentially have equally adverse effects on the performance of a PCR assay. Possible mechanisms for constraints limiting synonymous mutations may include overlapping reading frames, especially if those overlapping regions are functionally or structurally important. Identifying potential cold spots in the genome that are less likely to undergo synonymous substitutions is a significant challenge and a major obstacle to developing a robust PCR-based viral assay. Intra-species nucleotide alignment of available BKV sequences was followed by detailed intra- and interspecies nucleotide analysis of the target region to aid in designing assay primers and probe.

In the present work, the utilization of the intraspecies nucleotide alignment of BKV with the intraspecies nucleotide alignment of a closely related virus, JCV, for which a significant number of sequences were available, proved to be useful in substantiating that the Polyoma_4.2(f) and the $\mathrm{BKV}-\mathrm{MGB}$ probe binding regions within BKV was less likely to experience nucleotide sequence variation. The lack of polymorphisms at the corresponding positions in both BKV and JCV isolates provides stronger evidence for sequence conservation than does intra-species nucleotide analysis of BKV isolates alone. Conversely, the forward primer BKV_2.1(f) is reserved for use only as a secondary backup to Polyoma_4.2(f) due to the extent of polymorphisms seen among JCV isolates along the nucleotide stretch corresponding to the BKV_2.1(f) binding site.

Since the assay was first developed using $160 \mathrm{BKV}$ sequences, the number of available BKV complete genome sequences increased to 271 (Additional file 1). As presented in the results section, the increase in BKV sequence data did not demonstrate increased sequence variation in the region targeted by this analysis, validating the approach of incorporating the additional analyses discussed herein when designing a real-time PCR assay.

The amino acid and nucleotide sequence analyses that were performed identified the VP2 C-terminus as a probable functionally conserved region, consistent with the presence of an alpha-helix [18] and nuclear localization signal [19-22]. Targeting the VP2 gene for assay development is in contrast to the location of primers/ probes for most existing BKV assays [27]. Therefore this approach was successful in identifying a region different from those targeted by other assays that have become limited with the recognition of additional sequence variations.

Using functionally constrained regions for assay development might be expected to result in lack of specificity. However, sufficient nucleotide differences existed within the stable region to design an assay specific for $\mathrm{BKV}$. The three nucleotide difference between the BKV and JCV at the probe binding site was sufficient to meet assay specificity requirements by utilizing a Taqman MGB probe. By having the probe bind to the anti-sense strand, the respective positions of the inter-species nucleotide differences between the BKV and JCV were such that the minor groove binder of the Taqman ${ }^{\circ}$ MGB probe could be ideally positioned to provide a high degree of specificity. This configuration also provided the added benefit of having the probe bind to the same strand (anti-sense) as the BK virus specific reverse primer rather than on the sense strand that binds the Polyoma_4.2(f) primer, which amplifies both JCV and $\mathrm{BKV}$. It should be noted that the primer combination BKV_5.1(r) and Polyoma_4.2(f) is the only primer combination that has been designed and tested to perform under a BKV and JCV co-infection scenario.

Preliminary assay performance results indicate the BKV assay has a 6-log dynamic range, a lower detection limit of $1.5 \times 10^{1}$ copies/reaction and an intra-assay CV 
of $\leq 5.0 \%$. Good precision is important for distinguishing between assay variation and assay results that are associated with clinical treatment or indicative of the need for treatment. A wide dynamic range is significant for clinical application of the assay since the clinical need is for identification of patients with high viral load who may be at immediate risk for developing BKVAN and also for identification of low viral loads in patients being monitored for BKV reactivation. The assay also does not detect JCV and the presence of JCV does not inhibit detection of $\mathrm{BKV}$ at the concentrations tested, suggesting that accurate detection and quantitative results for BKV will be obtained for BKV clinical samples that are co-infected with JCV.

The relative lack of sequence variants in the VP2 gene suggests that an assay targeting this region will permit accurate detection of BKV and estimates of viral load regardless of subtype (Additional file 2). Such information is important in identifying active BKV infections in transplant recipients in time to initiate appropriate treatment. Further testing of this BKV assay is needed to fully characterize assay performance characteristics, clinical performance and to evaluate the impact of sequence variations.

\section{Conclusion}

Identifying stable PCR target regions when limited DNA sequence data is available is possible by combining multiple analysis techniques to elucidate genomic regions under functional and structural constraints. Applying this approach to the development of a real-time quantitative PCR assay for BKV resulted in an accurate method with potential clinical applications and advantages over existing BK assays.

\section{Methods}

\section{Sequence alignments: Amino Acid \& DNA}

A total of 185 amino acid sequences for the BKV VP2 gene were obtained from the NCBI database. The amino acid sequences of the VP2 genes for the other members of the family Polyomaviridae were collected for species in which the information was available. Multiple sequence alignment of the amino acid sequence of the VP2 gene was performed using ClustalW2 http://www.ebi.ac.uk.

At the time of preliminary assay development, a total of 160 nucleotide sequences of the Agno protein, 159 nucleotide sequences of the large- $T$ antigen, 157 nucleotide sequences of the small- $T$ antigen, and 159 nucleotide sequences of the three structural genes, VP1, VP2, and VP3 were obtained from the NCBI database http:// www.ncbi.nlm.nih.gov. Multiple alignments for the sequences of the six respective genes were performed using ClustalW2 http://www.ebi.ac.uk and MUSCLE http://www.ebi.ac.uk.
The entire amplicon sequence was input into the BLAST software http://www.ncbi.nlm.nih.gov to conduct species-specific searches and to analyze intra-species polymorphisms as well as interspecies sequence variation amongst closely related, clinically significant viruses. The BLAST search and results formatting were adjusted according to the number of available sequences within the database for each specific species. The results were formatted with inquiry sequence anchored with stars for identity to generate a multiple sequence alignment of the BLAST results.

\section{Oligonucleotides}

The theoretical Tm calculations as well as secondary structure and primer-dimer analysis for oligonucleotides were performed using OligoCalc [29] \& Primer3 [30]. The Tm under the actual reaction conditions was confirmed empirically. The need for a probe was confirmed by empirically testing for primer-dimer formation using the LightCycler ${ }^{\oplus} 480$ SYBR Green I Master reagent as well as post-amplification gel-electrophoresis.

Primers were purchased from Invitrogen Inc. (Carlsbad, CA). Two forward and two reverse primers were designed to amplify the C-terminus region of the VP2/3 gene. The BKV_5.1(r) comprised the primary reverse primer of the assay while the BKV_4.1(r) was utilized as an alternate. The reverse primer denoted as BKV_5.1(r) comprises the nucleotide sequence 5'-CTG CCC CTG GAC ACT CTC-3'. The reverse primer denoted as BKV_4.1(r) has the sequence 5'-TCA (I)AT GCT TCA AGA GCA GGT GT-3', wherein the nucleotide (I) represents deoxyinosine. The nucleotide sequence of the two forward primers denoted as Polyoma_4.2(f) and BKV_2.1 (f) are 5'-GCT CCT CAA TGG ATG TTG CCT-3' and 5'-CCC AGG AGG TGC TAA TCA AAG A-3', respectively. The Polyoma_4.2(f) comprised the primary forward primer of the assay while the BKV_2.1(f) was utilized as an alternate.

TaqMan $^{\oplus}$-MGB probes were supplied by Applied Biosystems Inc. (Foster City, CA). The BKV probe denoted as BKV-MGB probe (5'-6FAM-CCG TAC AGG CCT AGA A-MGB-NFQ-3') was tagged with 6 FAM $^{\mathrm{m}}$ at the 5 '-end and tagged with a MGBNFQ (Minor Groove Binder/Non-fluorescent quencher) at the 3'-end. The JCV probe denoted as JCV-MGB probe (5'-VIC-CGT ACA ACC CTA AAA G-MGB-NFQ-3') was tagged with VIC ${ }^{\oplus}$ at the 5 '-end and MGBNFQ (Minor Groove Binder/ Non-fluorescent quencher) at the 3'-end.

\section{Viral DNA Standards}

The BKV (strain MM) and JCV (strain MAD1) DNA standards were obtained from Advanced Biotechnologies Inc. (Columbia, MD), supplied at $1-2 \times 10^{5} \mathrm{DNA}$ copies/ $\mu \mathrm{l}$. Viral standard at $1.5 \times 10^{6} \mathrm{DNA}$ copies $/ \mu \mathrm{l}$ 
were produced from clinical $\mathrm{BKV}$ isolates using $\mathrm{TOPO}^{\circ}$ TA Cloning kit (Invitrogen Inc., CA, U.S.A.). Cloned viral DNA was quantified on the Roche LightCycler 480 using the commercially purchased standard.

\section{Quantitative real-time PCR}

Quantitative real-time PCR was performed on a Roche LightCycler ${ }^{\circ} 480$ instrument. PCR amplification was run in a final volume of $40 \mu \mathrm{l}$ per reaction comprising $30 \mu \mathrm{l}$ of PCR master mix and $10 \mu \mathrm{l}$ of sample, standard or PCR-grade water. PCR master mix for one reaction comprised of: $0.42 \mu \mathrm{M}$ forward primer, $0.42 \mu \mathrm{M}$ of reverse primer, $0.33 \mu \mathrm{M}$ of TaqMan ${ }^{\circ}$-MGB probe, and $20 \mu \mathrm{l}$ of LightCycler 480 Probes Master (2x).

Thermal cycling conditions when using the reverse primer BKV_4.1 were as follows: pre-incubation at $95^{\circ} \mathrm{C}$ for 10 minutes; 45 cycles of a three-step PCR wherein one cycle comprising of $95^{\circ} \mathrm{C}$ for 10 seconds (ramp rate $4.4^{\circ} \mathrm{C} / \mathrm{s}$ ), $60^{\circ} \mathrm{C}$ for 15 seconds (ramp rate $2.2^{\circ} \mathrm{C} / \mathrm{s}$ ) and $72^{\circ} \mathrm{C}$ for 1 second $\left(\right.$ ramp rate $4.4^{\circ} \mathrm{C} / \mathrm{s}$ ); and ending with a cool down step at $40^{\circ} \mathrm{C}$ for 30 seconds. Fluorescence was measured at each cycle following the third step at $72^{\circ} \mathrm{C}$.

Thermal cycling conditions when using the reverse primer BKV_5.1 were as follows: pre-incubation at $95^{\circ} \mathrm{C}$ for 10 minutes; 45 cycles of a three-step PCR wherein one cycle comprised $95^{\circ} \mathrm{C}$ for 10 seconds (ramp rate $4.4^{\circ}$ $\mathrm{C} / \mathrm{s}$ ), $58^{\circ} \mathrm{C}$ for 20 seconds (ramp rate $2.0^{\circ} \mathrm{C} / \mathrm{s}$ ) and $65^{\circ} \mathrm{C}$ for 1 second (ramp rate $4.4^{\circ} \mathrm{C} / \mathrm{s}$ ); and ending with a cool down step at $40^{\circ} \mathrm{C}$ for 30 seconds. Fluorescence was measured at each cycle following the third step at $65^{\circ} \mathrm{C}$.

BKV external standards at seven different concentrations $\left(10^{6}\right.$ copies $/ \mu \mathrm{l}, 10^{5}$ copies $/ \mu \mathrm{l}, 10^{4}$ copies $/ \mu \mathrm{l}, 10^{3}$ copies $/ \mu \mathrm{l}, 10^{2}$ copies $/ \mu \mathrm{l}, 10$ copies $/ \mu \mathrm{l}$ and $<10$ copies $/ \mu \mathrm{l}$ ) were included with each assay in order to generate the standard curve. Linear regression of the external standards and quantification of unknowns were performed using the LightCycler 480 software.

\section{Performance characterization of assay}

Specificity testing. Specificity data were generated by testing analytical samples containing BKV, JCV and mixed samples containing both BKV and JCV. Serial dilutions of BKV to result in from $1.5 \times 10^{1}$ copies/reaction to $1.5 \times 10^{7}$ copies/reaction were tested individually ( 2 replicates of each concentration) and in the presence of $7 \times 10^{3}$ copies/reaction of JCV (10 replicates of each concentration). JCV at $1.4 \times 10^{4}$ copies/reaction (10 replicates) was also tested individually. A total of 94 specificity samples were tested. Five replicates of a negative control were also tested.

Analytical sensitivity evaluation. Analytical sensitivity samples comprised of BKV at concentrations of 150, 75, 15 and 1.5 copies/reaction were used to evaluate assay sensitivity. The BKV samples were prepared by serial dilution of a BKV standard. A total of 120 samples, 10 replicates of each concentration with 3 primer combinations, were tested in the assay. Five replicates of a negative control were also tested.

Assay Precision. The intra-assay precision of the BKV assay was evaluated. The samples were constructed by serial dilution of BKV standard and contained from 1.5 $\times 10^{1}$ copies/reaction to $1.5 \times 10^{7}$ copies/reaction. Multiple replicates (10) of the seven different concentrations of the BKV standard were tested with 3 primer combinations in one run and the variance in the $\mathrm{Cp}$ (crossing point) calculated to determine the reproducibility of the assay. A total of 210 samples were tested. Five replicates of a negative control were also tested.

\section{Additional material}

Additional file 1: Subtypes and GenBank accession numbers used in this study. Accession numbers and subtype identity of the $271 \mathrm{BKV}$ sequences used in this study are presented. (A) Subtype I accounted for 70\% (190/271), (B) Subtype II accounted for <2\% (4/271), (C) Subtype III accounted for $<2 \%$ (4/271), and (D) Subtype IV accounted for 27\% (73/ 271).

Additional file 2: Alignment of 271 BKV sequences corresponding to the VP2(VP3) C-terminus region. Nucleotide alignment of $271 \mathrm{BKV}$ sequences obtained from GenBank aligned to BKV Dunlop reference strain (V01108.1; nucleotides 1437 to 1679) is shown. The nucleotide range for each individual sequences are provided. Partial VP1 sequences were excluded from the alignment. The positions of the primers (underlined) and probe (boxed) are indicated on the BKV reference sequence. The subtype and subgroup identity for each sequence is provided. Subtypes were determined using the typing schema established by Jin et al. [31] and by Luo et al. [32]. The results were confirmed through phylogenetic analyses of the VP1 gene (data not shown). Subgroup identities for the 271 sequences were confirmed through phylogenetic analyses and trees constructed from whole genome sequences as previously described [31,33]. Phylogenetic trees were visualized using MEGA version 4 [34].

\section{Acknowledgements}

We thank Mary Kay Romero for writing services on behalf of Synteract, Inc.

\section{Author details}

${ }^{1}$ Metic Transplantation Laboratory, USC, Keck School of Medicine, LoS Angeles, CA, USA. ${ }^{2}$ Transplant Immunology Laboratory, Cedars-Sinai Medical Center, Los Angeles, CA, USA. ${ }^{3}$ Division of Transplantation, Department of Surgery, Loma Linda University Medical Center, Loma Linda, CA, US. ${ }^{4}$ Department of Pathology, Loma Linda University Medical Center, Loma Linda, CA, US. ${ }^{5}$ Internal Medicine, USC, Keck School of Medicine, Los Angeles, CA, US.

\section{Authors' contributions}

$\mathrm{KKI}$ carried out the assay design, sequence alignment and assay performance studies. SHQ, YM, DZ, MEM and JG-G assisted with the assay optimization and cloning. MS and YQ participated in acquiring the samples. MT participated in assay validation. $K M, Y Q$, and $Y$ I participated in the conception and design of the studies. AK reviewed patient charts and confirmed clinical diagnoses. WTS reviewed histopathological findings to confirm clinical diagnoses. DDI reviewed test results and conducted further blind testing using this diagnosing system. All authors read and approved the final manuscript. 


\section{Competing interests}

The primary author is listed as an inventor in a published International Patent Application filed by Metic Immunogenetic Consultant, Inc., entitled "Detection of Polyomavirus".

Received: 31 May 2010 Accepted: 29 October 2010

Published: 29 October 2010

\section{References}

1. Knowles WA: Discovery and epidemiology of the human polyomaviruses BK virus (BKV) and JC virus (JCV). Adv Exp Med Biol 2006, 577:19-45.

2. Lundstig A, Dillner J: Serological diagnosis of human polyomavirus infection. Adv Exp Med Biol 2006, 577:96-101.

3. Khalili K, Stoner GL: Human polyomaviruses: molecular and clinical perspectives. 1 edition. New York: Wiley; 2001.

4. Kwak EJ, Vilchez RA, Randhawa P, Shapiro R, Butel JS, Kusne S: Pathogenesis and management of polyomavirus infection in transplant recipients. Clin Infect Dis 2002, 35:1081-1087.

5. Hirsch HH, Brennan DC, Drachenberg CB, Ginevri F, Gordon J, Limaye AP, Mihatsch MJ, Nickeleit V, Ramos E, Randhawa P, et al: Polyomavirusassociated nephropathy in renal transplantation: interdisciplinary analyses and recommendations. Transplantation 2005, 79:1277-1286.

6. Schwarz A, Mengel $M$, Haller $H$, Niedermeyer J: Polyoma virus nephropathy in native kidneys after lung transplantation. $A m J$ Transplant 2005, 5:2582-2585.

7. Limaye AP, Smith KD, Cook L, Groom DA, Hunt NC, Jerome KR, Boeckh M: Polyomavirus nephropathy in native kidneys of non-renal transplant recipients. Am J Transplant 2005, 5:614-620.

8. Randhawa P, Vats A, Shapiro R: The pathobiology of polyomavirus infection in man. Adv Exp Med Biol 2006, 577:148-159.

9. Munoz P, Fogeda M, Bouza E, Verde E, Palomo J, Banares R: Prevalence of $B K$ virus replication among recipients of solid organ transplants. Clin Infect Dis 2005, 41:1720-1725.

10. Thomas L, Vilchez R, Milstone A, Butel J, Dummer S: Polyomavirus infection in lung transplant recipients [Abstract \#633]. 42nd Meeting of the Infectious Diseases Society of America 2004, 165.

11. Drachenberg CB, Beskow CO, Cangro CB, Bourquin PM, Simsir A, Fink J, Weir MR, Klassen DK, Bartlett ST, Papadimitriou JC: Human polyoma virus in renal allograft biopsies: morphological findings and correlation with urine cytology. Hum Pathol 1999, 30:970-977.

12. Hirsch HH, Knowles W, Dickenmann M, Passweg J, Klimkait T, Mihatsch MJ, Steiger J: Prospective study of polyomavirus type BK replication and nephropathy in renal-transplant recipients. N Engl J Med 2002, 347:488-496

13. Nickeleit V, Klimkait T, Binet IF, Dalquen P, Del Zenero V, Thiel G, Mihatsch MJ, Hirsch $\mathrm{HH}$ : Testing for polyomavirus type BK DNA in plasma to identify renal-allograft recipients with viral nephropathy. $N$ Engl J Med 2000, 342:1309-1315.

14. Kadambi PV, Josephson MA, Williams J, Corey L, Jerome KR, Meehan SM, Limaye AP: Treatment of refractory BK virus-associated nephropathy with cidofovir. Am J Transplant 2003, 3:186-191.

15. Vats A, Shapiro R, Singh Randhawa P, Scantlebury V, Tuzuner A, Saxena M Moritz ML, Beattie TJ, Gonwa T, Green MD, Ellis D: Quantitative viral load monitoring and cidofovir therapy for the management of BK virusassociated nephropathy in children and adults. Transplantation 2003, 75:105-112.

16. Larkin MA, Blackshields G, Brown NP, Chenna R, McGettigan PA, McWilliam H, Valentin F, Wallace IM, Wilm A, Lopez R, et al: Clustal W and Clustal X version 2.0. Bioinformatics 2007, 23:2947-2948.

17. Edgar RC: MUSCLE: multiple sequence alignment with high accuracy and high throughput. Nucleic Acids Res 2004, 32:1792-1797.

18. Chen XS, Stehle T, Harrison SC: Interaction of polyomavirus internal protein VP2 with the major capsid protein VP1 and implications for participation of VP2 in viral entry. EMBO J 1998, 17:3233-3240.

19. Chen $L F$, Ito $K$, Murakami $Y$, Ito $Y$ : The capacity of polyomavirus enhancer binding protein 2alphaB (AML1/Cbfa2) to stimulate polyomavirus DNA replication is related to its affinity for the nuclear matrix. Mol Cell Biol 1998, 18:4165-4176.

20. Ishii N, Nakanishi A, Yamada M, Macalalad MH, Kasamatsu H: Functional complementation of nuclear targeting-defective mutants of simian virus 40 structural proteins. J Virol 1994, 68:8209-8216.
21. Chang D, Haynes JI, Brady JN, Consigli RA: Identification of a nuclear localization sequence in the polyomavirus capsid protein VP2. Virology 1992, 191:978-983

22. Shishido-Hara $Y$, Ichinose $S$, Higuchi $K$, Hara $Y$, Yasui K: Major and minor capsid proteins of human polyomavirus $\mathrm{JC}$ cooperatively accumulate to nuclear domain 10 for assembly into virions. J Virol 2004, 78:9890-9903.

23. Clever J, Dean DA, Kasamatsu $\mathrm{H}$ : Identification of a DNA binding domain in simian virus 40 capsid proteins Vp2 and Vp3. J Biol Chem 1993, 268:20877-20883.

24. The UniProt Consortium: The Universal Protein Resource (UniProt); SwissProt Accession \#P03094. 2010 [http://www.uniprot.org/uniprot/P03094]

25. Seif I, Khoury G, Dhar R: The genome of human papovavirus BKV. Cell 1979, 18:963-977.

26. Yang $\mathrm{RC}$, Wu R: BK virus DNA: complete nucleotide sequence of a human tumor virus. Science 1979, 206:456-462.

27. Hoffman NG, Cook L, Atienza EE, Limaye AP, Jerome KR: Marked variability of BK virus load measurement using quantitative real-time PCR among commonly used assays. J Clin Microbiol 2008, 46:2671-2680.

28. Limaye AP, Jerome KR, Kuhr CS, Ferrenberg J, Huang ML, Davis CL, Corey L, Marsh CL: Quantitation of BK virus load in serum for the diagnosis of BK virus-associated nephropathy in renal transplant recipients. J Infect Dis 2001, 183:1669-1672

29. Kibbe WA: OligoCalc: an online oligonucleotide properties calculator. Nucleic Acids Res 2007, 35:W43-46.

30. Rozen S, Skaletsky H: "Primer3 on the www for general users and for biologist programmers.". In Bioinformatics Methods and Protocols: Methods in Molecular Biology. Edited by: Krawetz S, Misener S. Totowa, NJ: Humana Press; 2000:365-386[http://fokker.wi.mit.edu/primer3/].

31. Jin L, Gibson PE, Booth JC, Clewley JP: Genomic Typing of BK Virus in Clinical Specimens by Direct Sequencing of Polymerase Chain Reaction Products. J Med Virol 1993, 41:11-17.

32. Luo C, Bueno M, Kant J, Martinson J, Randhawa P: Genotying Schemes for Polyomavirus BK, Using Gene-Specific Phylogenetic Trees and Single Nucleotide Polymorphism Analysis. J Virol 2009, 83:2285-2297.

33. Sharma PM, Gupta G, Vats A, Shapiro R, Randhawa P: Phylogenetic Analysis of Polyomavirus BK Sequences. J Virol 2006, 80:8869-8879.

34. Tamura K, Dudley J, Nei M, Kumar S: MEGA4: Molecular Evolutionary Genetics Analysis (MEGA) Software Version 4.0. Mol Biol Evol 2007. 24:1596-1599.

doi:10.1186/1743-422X-7-295

Cite this article as: Iwaki et al.: Development of a real-time quantitative PCR assay for detection of a stable genomic region of BK virus. Virology Journal 2010 7:295.

\section{Submit your next manuscript to BioMed Central and take full advantage of:}

- Convenient online submission

- Thorough peer review

- No space constraints or color figure charges

- Immediate publication on acceptance

- Inclusion in PubMed, CAS, Scopus and Google Scholar

- Research which is freely available for redistribution 